\title{
IoT- Based Traffic Signal Control for Ambulance
}

\author{
Reeta R., Kirithiga R., Kavitha V. Kumar, Jaishree M.
}

\begin{abstract}
In modern era, due to increase in traffic in the city, emergency vehicles take more time to reach the destination. The current, time-based traffic management system is not suitable and also not flexible for present day traffic, especially at the intersection where the traffic needs to be controlled for vehicles from all four directions. To solve this problem, we bring users a sound detector with automatic recording of various vehicle sounds and distinguishing the presence of ambulance in a particular lane by detecting the siren sound. The captured ambulance sound is processed using IOT and sent to the traffic pole to enhance the traffic clearance. This is carried out by placing the sensors in each lane and a sensor near the traffic pole to indicate that the ambulance has crossed the lane. In this method the traffic signal controller decides when the vehicle has to cross the road and also provide importance to the emergency vehicle.
\end{abstract}

Keywords: sound sensors, IOT, Arduino UNO, traffic light, Ambulance

\section{INTRODUCTION}

Internet of Things is a giant network with connected devices and sensors that gather and this is used to share data about how they are used and the environment in which they are operated. IOT is the collection of smart things which transmit and receive data in a much secured manner $[8,2]$. Sensors are embedded in every physical device. These sensors continuously emit data of the working state of the devices. Sensors are functionally simple devices that convert physical variables into electrical signals. Smart sensors [8] are built as IOT components that change the existing variable that is being measured into a digital data stream for transmission from one gateway to another. Traffic is generally organized in many domains with marked lanes, junctions, intersections, traffic signals, or signs. The main problem is to regulate the movements at an intersection.

When properly timed and maintained, a traffic signal increases the traffic handling capacity of an intersection. In

Revised Manuscript Received on February 05, 2020.

* Correspondence Author

Reeta R.,* Department of Computer Science and Engineering, Rajalakshmi Engineering College, Chennai, Tamil Nadu, India.

E-mail: reeta.r@rajalakshmi.edu.in Rajalakshmi Engineering College, Chennai, Tamil Nadu, India.

E-mail: kirithiga.r.3198@gmail.com

Kavitha V. Kumar, Department of Computer Science and Engineering,

Rajalakshmi Engineering College, Chennai, Tamil Nadu, India.

E-mail: kavithavijay498@gmail.com

Jaishree M., Department of Computer Science and Engineering,

Rajalakshmi Engineering College, Chennai, Tamil Nadu, India.

E-mail: jaimugil48@gmail.com

(C) The Authors. Published by Blue Eyes Intelligence Engineering and Sciences Publication (BEIESP). This is an open access article under the CC BY-NC-ND license (http://creativecommons.org/licenses/by-nc-nd/4.0/)
Kirithiga R., Department of Computer Science and Engineering,

most cases, the intersections are the limit to maximum throughput of the roadway. That is, increasing the number of lanes or the speed limit will not have any effect on the overall capacity of the road. The only way to increase the number of vehicles that travels safely from one point to another is to increase the efficiency of the intersections. Sirens are used function of any traffic signal is to assign right way to allow emergency vehicles such as ambulances, police cars, and fire trucks. The traffic analysis in $[1,6,11]$ is based on selection of vehicles by photoelectric sensors, radio frequency identification and GPS. Adaptive traffic signal control [4] method considers a number of factors on traffic to determine green light sequence and the optimal green light length. A siren is a warning device which makes a lengthy, loud sound, thus alerting the vehicles on the way. Sirens are integrated into a warning system such that it is linked with other warning media. Siren comprises of multiple tones in which each tone has its own meaning given to alert the residents of the impending danger. A siren sound in emergency vehicles indicates that the direction in which it is approaching. It is done using varying the pitch of the sound. An ambulance is an emergency vehicle [7] to provide emergency care to sick or injured people and to get them to hospital. Ambulance is mainly used to transport patients between hospitals. The siren sound of the ambulance has an alternative sequence of high frequency and a low frequency signal, the sequence of the frequency change is used to be the feature of the sound. They provide easy and quick access to health services, particularly out of hours. Thus it is very important for the ambulance to reach the destination at correct time. Traffic jams are common due to heavy traffic on road, as a result the emergency vehicles like ambulance and fire engines get stuck in traffic. Android mobile can be used to send message to the traffic controller using software installed in it [7]. The message is sent through the short message service available in phone to the GSM module. Image processing is a widely used method for identifying the lane in which the ambulance is approaching. The proposed method will be useful for the ambulance to pass through the traffic junctions without waiting, so that they can reach their destination quickly. GPS [3] does not help in recognizing and prioritizing emergency vehicles, its main function is to maintain the traffic system. The proposed method will be beneficial for the vehicles at emergency circumstances to pass through the traffic junctions without waiting so that they can reach their destination on time.

\section{LITERATURE SURVEY}

The author in [1] concentrates on providing a clear design of an integrated intelligent system for management and controlling traffic lights supported distributed in a lengthy range using Photoelectric 


\section{IoT- Based Traffic Signal Control for Ambulance}

Sensors in distances before and after the traffic lights by appropriate selection of vehicle. The main problem is to reduce the processing time and to enable message scheduling and sending in an optimal way. It is based on sending or receiving messages through any of the IOT devices.

Delay-Aware Accident Detection and Response System provides an improvement by making efficient use of the benefits of sophisticated features of smart phones and fog computing to propose and develop a low-cost and delay-aware accident detection and response system, which we term Emergency Response and Disaster Management System (ERDMS) using GPS and android phone. The problem to detect the distance of the vehicles from a specific location, and signal is addressed. [3]

Adaptive traffic signal control method considers a number of factors on traffic to determine green light sequence and the optimal green light length. Green light sequence, using the traffic data real-time is determined using decision making method. The methods used involve vehicle detection, green light sequence determination and light length determination. The distance travelled by the green light is determined by the light length. [4]

The authors in [5] described that sensors can be used for measurement of traffic density. Traffic lights will manage data about the various traffic density based on the data collected particularly at junction, supported the density of the traffic which is measured using RFID technique. However, using this technique it is not possible to identify the density of the traffic beyond a particular distance and it is too expensive. Similar method is employed in adaptive traffic control [6].

IOT Based Traffic Signal Control Technique for Helping Emergency Vehicles [7] is proposed for the better management of the traffic of emergency vehicles through the use of internet of things (IOT). The proposed method enables the emergency vehicles to send message to the traffic signal controller placed at the traffic junction where two or more lanes meet regarding their arrival in order that the traffic can be regulated accordingly. In this method the user travelling in the ambulance carries the android phone with the software deployed in it. The SMS (Short Messaging Service) available in the mobile phone of the users , is used to send the messages to the GSM module.

The author in [9] proposed the process of synchronization and connected vehicle technology where each vehicle interacts with each other. This method displays the results comparing with the existing traffic control system. This technology comprises of connected vehicles, process synchronization, intelligent transportation system, real-time processing and intersection management.

This paper has introduced a new algorithm to avoid the traffic deadlock. It also depicts the singe intersection model for simulation, which represents the effective results and the flexibility for the development.

Traffic congestion is a serious problem in big cities where the population is more. In this paper [11] the traffic is controlled using GPS which is used to detect the position of the moving cars. IOT is installed in the moving car which is guided by GPS. The traffic analysis helps the car to take the best route without spending much time in traffic.

The transport network consists of heterogeneous stochastic agents such as intersection stochastic agents such as intersection controllers and road users. The task of adaptive Traffic Light Control is extremely challenging. In this paper
[12] the author has analysed the complexity of Traffic Light Control on a single isolated intersection and used the concept of supervised learning. The bottle neck of this method is the data generation and annotation on the growing data in the intersection.

Iman Askerzade, Mustafa Mahmood introduced a system to control the extension time of traffic light. The extended time of traffic light is implemented in single junction based on Fuzzy Logic. This method also shows the simulation results obtained which also marks its better performance and is cost effective. The performance here is calculated by comparing the fuzzy logic with the fixed time controllers. Fuel, Air and Noise pollution are reduced by minimisation in the waiting time. This model can also be developed for better usage in future. [13]

Darwish T, Bakar K A have proposed a system on Traffic density estimation in vehicular ad hoc networks (VANETs) . The main reason for proposing this system is to safeguard traffic, distribution of data, and traffic management. Technologies related to wireless communication in vehicles are rapidly increasing and estimation on real-time infrastructure density free methods are growing day by day. Algorithms in this system is used in both infrastructure based and infrastructure free density method. [14]

\section{ARCHITECTURE}

The architecture diagram shows various modules involved in the project design:

This architecture diagram is a pictorial representation of our proposed system in which, two sound sensors, bread board and an Arduino UNO R3 kit is used. One sound sensor is placed at a distance of $120 \mathrm{~m}$ from the traffic light and other placed near the traffic pole to sense the siren sound. Traffic light control is wired on the bread board.

The sound sensor is used to predict the distance of the the vehicles to and from it. In our system , one sound sensor is placed at a distance of $120 \mathrm{~m}$ from the traffic pole and the other is placed near the traffic pole. A sound sensor is used to detect the sound of an ambulance, which is transmitted to the traffic pole for light change. The traffic light control is wired on a bread board to make changes in the lights.

All these components are connected together using an Arduino UNO kit, that is an open source microcontroller board equipped with digital and analog input and output pins. The kit is programmable using Arduino IDE and can be powered using an USB cable or connecting it externally to a 9V battery. 


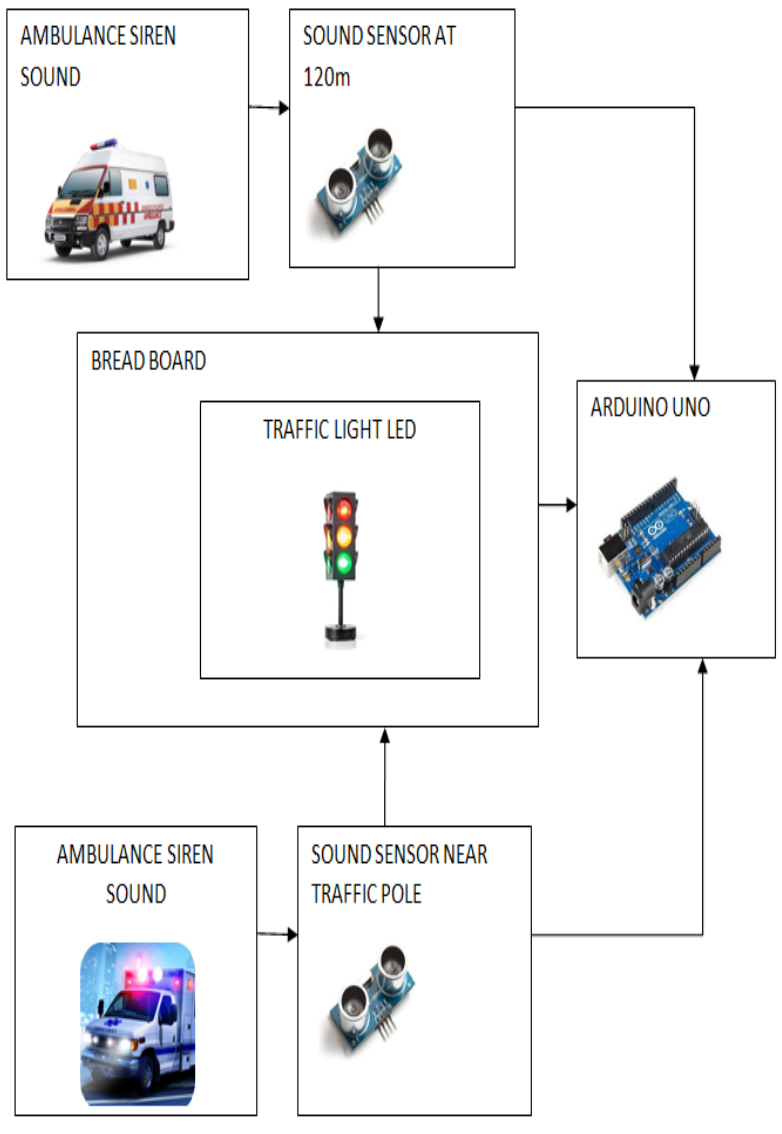

Fig 1. Model Architecture

\section{WORKING MODULES}

Initial Detection of Ambulance Sound:

When the ambulance passes by the lane and is at a distance of $120 \mathrm{~m}$, the sound sensor detects the ambulance by recognising its sound level which is $120 \mathrm{~dB}$.

\section{Transmitting signal:}

The detected sound is then transmitted by the sound sensor to the traffic controller which is located at the intersection. This in turn enables them to change the signal at correct time.

\section{Switching the lights at the time of ambulance crossing:}

The traffic light at a particular lane in which the ambulance passes is changed to green and all other lanes are changed to red, thus allowing the uninterrupted passage of ambulance.

\section{Detecting ambulance sound near traffic pole:}

When the ambulance crosses the lane and reaches the traffic pole, the sound sensor detects the ambulance by recognising its frequency.

\section{Directing signal after the ambulance crosses:}

The detected sound is now transmitted by the sound sensor to the traffic controller again. This in turn enables them to change the signal. The traffic light at all the lanes meeting at the junction is changed back to normal form, thus allowing flow of vehicles in all the lanes.

\section{Reverting back the changes made:}

The traffic light is changed to normal form, thus allowing flow of vehicles in all the lanes.

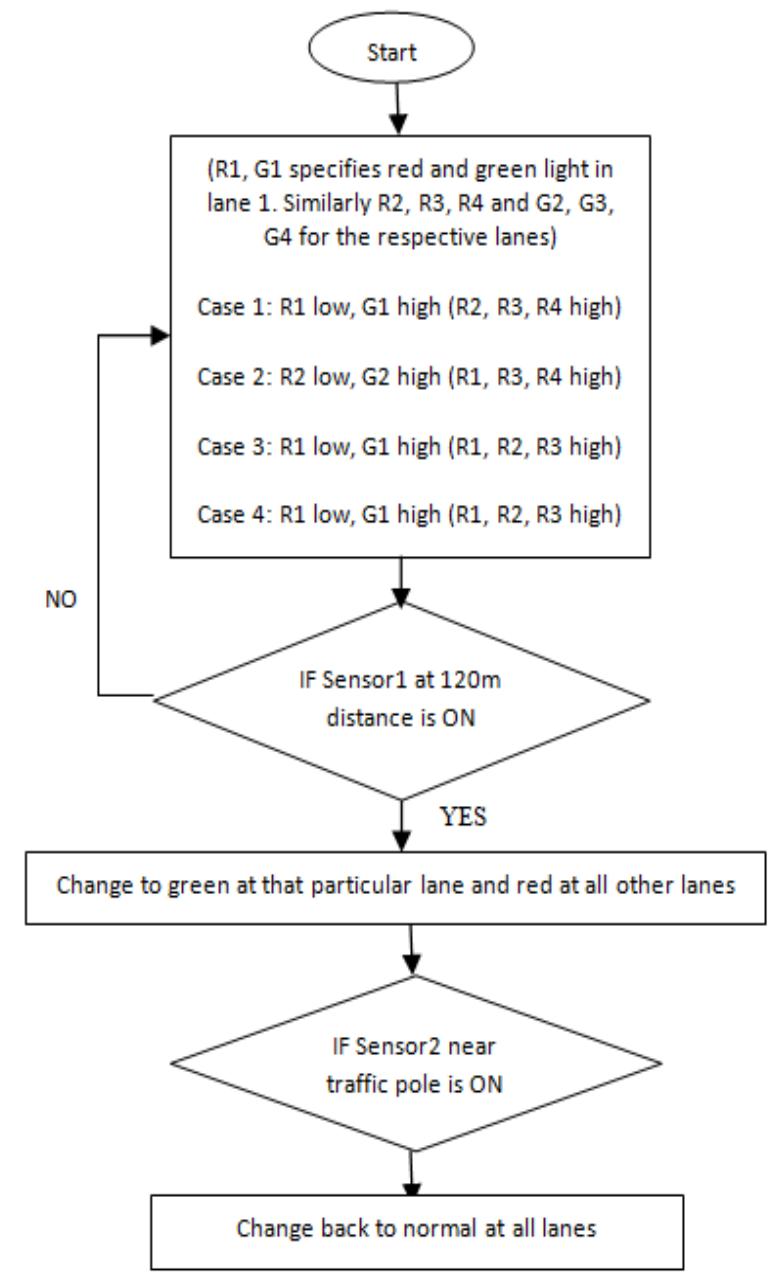

Fig 2: Workflow of IOT based traffic control system

\section{RESULTS}

The aim of this design is to obtain a highly accurate model for recognising the ambulance by detecting the siren sound at all the lanes. This is deployed by making use of sound sensor at a distance of $120 \mathrm{~m}$ and the other sensor near the traffic pole. The sound sensor detects the ambulance sound passing the lane at $120 \mathrm{~m}$ distance. This detected sound is sent to the traffic controller.

The traffic light at that particular lane is changed to green and all other lanes are changed to red using Arduino UNO. After the ambulance crosses the lane, the sensor placed near the traffic pole detects the ambulance sound, and concludes that the ambulance has passed and thus sends the message to the traffic controller to change the traffic light back to normal at all the lanes. The efficiency of using the sound sensor over other method of traffic clearance such as Image processing, Android, GPS are analysed. The given analysis on traffic signal control for ambulance, travelling a distance of $50 \mathrm{Km}$ using GPS, image processing, android software and IOT shows that, the average time taken to reach the destination using GPS is 30 minutes, image processing is 26 minutes, android software is 22 minutes and IOT is 20 minutes. Thus, IOT seems to be an efficient way to reduce the delay of ambulance.

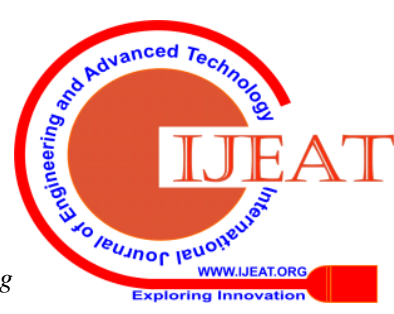




\section{IoT- Based Traffic Signal Control for Ambulance}

\begin{tabular}{|c|c|}
\hline METHODS & $\begin{array}{c}\text { TIME TO REACH } \\
\text { THE HOSPITAL } \\
\text { IN MINUTES }\end{array}$ \\
\hline GPS & 30 \\
\hline $\begin{array}{c}\text { IMAGE } \\
\text { PROCESSING }\end{array}$ & 26 \\
\hline $\begin{array}{c}\text { ANDROID } \\
\text { SOFTWARE }\end{array}$ & 22 \\
\hline IOT & 20 \\
\hline
\end{tabular}

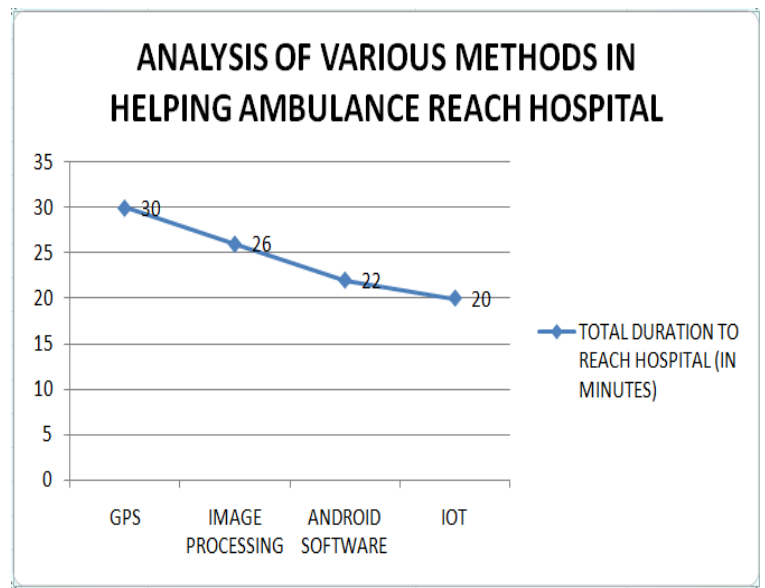

Fig 3: Analysis of various methods in helping reach hospital

\section{CONCLUSION}

As human life is very valuable, people should be conscious and follow the security measures. In this paper, we have presented an efficient traffic signal system by which, we will manage the ambulance to succeed in the destination by avoiding much traffic. Our system is meant to realize lower vehicular waiting time than the prevailing ones. The most contribution of our proposed system is that, it is often used to select the simplest possible options for changing the green light and control the traffic system, such that the waiting time is minimized. Moreover, the implementation cost is reduced, because it does not involve any complex hardware installation. The plan was developed to regulate two or four way traffic junction. Additionally, our plan can be extended to handle various lanes and assigning priority to the ambulance

if the ambulances are approaching at different lanes simultaneously. This IOT and sensor based system was developed to provide automated environment and save human lives.

\section{REFERENCES}

1. Ahmed S. Salama, Bahaa K. Saleh, Mohamad M. Eassa(2016, Nov) Intelligent Cross Road Traffic Management System (ICRTMS) Presented at 2 nd International Conference on Computer Technology and Development (ICCTD 2016).

2. Arnab Kumar Saha, Sachet Sircar, Priyasha Chatterjee, Souvik Dutta, Anwesha Mitra, Aiswarya Chatterjee, Soummyo Priyo Chattopadhyay, Himadri Nath Saha. Department of Computer Science and Engineering IEEE 2018. A Raspberry Pi Controlled Cloud Based Air and Sound Pollution Monitoring System with Temperature and Humidity Sensing.

3. Bilal Khalid dar1, Munam Ali Shah 1, Saif Ul Islam 2, Castren Maple 3, Shafaq Mussadiq 4, and Zuleman Khan 5 ,February 20, 2019, accepted March 11, 2019, date of publication May 1, 2019, date of current version June 11, 2019, Delay-Aware Accident Detection and Response System Using Fog Computing.

4. Binbin Zhou, Dept. of Comput., Hong Kong Polytech. Univ., Hong Kong, China Jiannong Cao,Dept. of Comput., Hong Kong Polytech. Univ., Hong Kong, China, Xiaoqin Zeng, Dept. of Computer Science \& Technol., Hohai Univ.,China, Hejun Wu, Dept. of Computer
Science, Sun Yat-sen Univ., Guangzhou, China, "Adaptive Traffic Light Control in Wireless Sensor Network-Based Intelligent Transportation System".

5. Lobna M. Eslim Hossam, S. Hassanein, Walid M. Ibrahim, Abdallah Alma'aitah .," A cooperative localization scheme using RFID crowdsourcing and time-shifted multilateration", in LCN , 2016 IEEE $39^{\text {th }}$ Conference, Sept .

6. Sakhawat Hossan, Naushin Nower, Institute of Information Technology, University of Dhaka, Efficient Dynamic Traffic Light Control for ITS, ICC' 17, March 22 2017, Cambridge, United Kingdom, 2017 ACM.

7. Sneha Tammishetty, T.Ragunathan, Sudheer Kumar Battula, B.Varsha Rani, P. Ravi Babu, Raghu Ram Reddy Nagireddy, Vedika Jorika and V. Maheshwar Reddy, IOT Based Traffic Signal Control Technique for Helping Emergency Vehicles Springer Science and Bussiness Media Singapore 2017.

8. Zanella Andrea, Nicola Bui, Angelo Castellani, Vangelista Lorenzo, Michele Zorzi, "Internet of Things for Smart Cities," inInternet of Things Journal, IEEE, vol.1, no.1, Feb. 2014.

9. Waris Hooda, Amogh Bhole, Pradeep Kumar Yadav and Deptii D.Chaudhari, International Institute of Information Technology, ICTCS'16, ACM 2016, An Image Processing Approach to Intelligent Traffic Management System.

10.Khac-Hoai Nam Bui, O-Joun Lee, Jason J. Jung and David Camacho, Dynamic Traffic Light Control System Based on Process Synchronization Among Connected Vehicles, Department of Computer Science and Engineering, Springer International Publishing Switzerland 2016.

11.Linna Wu, Huan Li, Feng Ren, Lizhuo Zhang, IoT-enabled traffic analysis, Proceedings of International Conference on Internet of Things Design and Implementation ACM, April 2019.

12. Gulnur Tolebi, Daniyar KurmanKhojayev, Nurlan S.Dairbekov, Proceedings of $5^{\text {th }}$ International Conference on Engineering and MIS, Road traffic demand estimation and traffic signal control, ACM, June 2019.

13.Iman Askerzade, Mustafa Mahmood, 2010. Control the Extension Time of Traffic Light in Single Junction by Using Fuzzy Logic. Departmen of computer Engineering, University of Ankara, Turkey, IEEE.

14.Darwish T, Bakar K A. Traffic density estimation in vehicular ad hoc networks : a review. Ad Hoc Networks, 2015, 24(PA): 337-351, IEEE.

15.Omkar Udawant, Nikhil Thombare, Devanand Chauhan, Akash Hadke, Dattatray Waghole ,Smart Ambulance System using IoT, International Conference on Big Data, ToT and Data Science 2017, IEEE.

16.P Manikanta, Saddam Hussian SK, R Tamil Kodi, ,Iot Ambulance With Automatic Traffic Light Control, 2019 International Conference on Vision towards Emerging Trends in Communication and Networking, IEEE.

17.Reeta R, Pavithra G, Priyanka V, Raghul JS, Department of Computer Science and Engineering, Rajalakshmi Engineering College, An Empirical Investigation on Hazardous Gas Detection, International Journal of Applied Engineering Research ISSN 0973-4562 Volume 13, Number 9 (2018).

18.Reeta R, Pushpavathi V, Sanchana R, Shanmugapriya V, Department of Computer Science and Engineering, Rajalakshmi Engineering College, Chennai, India, International Journal of Applied Engineering Research, Volume 13, 2018

\section{AUTHORS PROFILE}

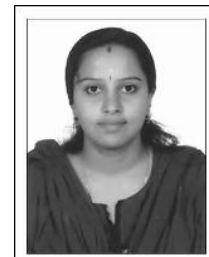

Mrs Reeta R., pursed Bachelor of Computer Science and Engineering in C.S.I College of Engineering, Anna University in 2004 and Master of Software Engineering in Easwari Engineering College, Anna University at 2012 and currently working as Assistant Professor in Department of Computer Science and Engineering at Rajalakshmi Engineering College. She has published 8 research papers in reputed International and national journals, which includes IEEE and Scopus indexed journal and it is also available online. Her main research work focuses on IoT, Data Mining, Software Engineering and Software Testing. She has 5 years of Teaching experience. Received the best faculty award in Computer Science and Engineering. 


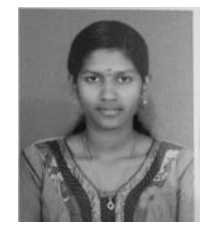

Kirithiga R., is currently pursuing her Bachelor of Engineering degree in Computer Science and Engineering at Rajalakshmi Engineering College, Anna University. She has completed 3 Mini projects on HTML, CSS and javascript with database connectivity. Her areas of interest include Python, HTML, CSS, javascript, Java, DBMS and IoT. She is currently placed in TCS Digital. She has presented paper on Block chain technology at Meenakshi Sundararajan Engineering College. She has completed NPTEL certification course on Python and Introduction to Modern Application and development. She has completed modules on python, $\mathrm{C}$ and algorithms in HackerRank and has secured a rank of top $4 \%$ in python programming.

Kavitha V. Kumar, is currently pursuing Bachelor of Engineering degree in Computer Science and Engineering. She has completed three mini projects Restaurant Management System using the POS system, Hospital Management System using JavaScript and Database Connectivity, Online Quiz App which was implemented using Mobile Application Development. She is doing her Internship in Concinnate partners, where she got placed through Campus Interview. Her area of interest is Cloud Computing, HTML, CSS and javascript. She has also been certified in Pega CSA(V7.4). She has been certified in Java and BigData. Her content writing for best entertainer has been published in a Magazine named "PUNNAGAI ULAGAM" 2013 Edition.

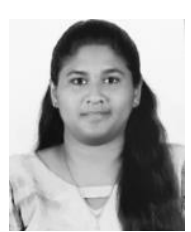

M Jaishree, is currently pursuing Bachelor of Engineering degree in Computer Science and Engineering. She has completed 3 Mini projects using HTML, CSS, javascript with database connectivity and online application development. Her areas of interest include HTML, CSS, javascript, java and Dot Net. She is currently placed in Avasoft. She has completed her internship for duration of one month. She has been certified in Java and BigData. She has participated in ZOHO workshop for python development. She has completed CSA(V7.4) certification in pega technologies. C has worked in Dot net and C sharp project. She has also participated in workshop of gaming and hacking. 